\title{
The MATRICS Consensus Cognitive Battery in Patients with Bipolar I Disorder
}

\author{
Katherine E Burdick*, 1,2,3, Terry E Goldberg 1,2,3, Barbara A Cornblatt ${ }^{1,2,3}$, Richard S Keefe ${ }^{4}$, Chaya B Gopin', \\ Pamela DeRosse', Raphael J Braga' and Anil K Malhotra ${ }^{1,2,3}$ \\ 'Department of Psychiatry, The Zucker Hillside Hospital; North Shore-Long Island Jewish Health System, Glen Oaks, NY, USA; ${ }^{2}$ Department of \\ Psychiatry, Albert Einstein College of Medicine, Bronx, NY, USA; ${ }^{3}$ Center for Psychiatric Neuroscience, The Feinstein Institute for Medical \\ Research, Manhasset, NY, USA; ${ }^{4}$ Department of Psychiatry, Duke University, Durham, NC, USA
}

\begin{abstract}
The Measurement and Treatment Research to Improve Cognition in Schizophrenia (MATRICS) initiative was devised to identify a neurocognitive battery to be used in clinical trials targeting cognition in schizophrenia, a process, which resulted in the MATRICS Consensus Cognitive Battery (MCCB). The MCCB has been selected by the United States Food and Drug Administration to be used as the primary outcome measure in registry trials for cognitive agents in schizophrenia. Given the clinical and cognitive overlap between schizophrenia and bipolar disorder (BPD), it is likely that any compound shown to have cognitive benefits in schizophrenia will subsequently be tested in BPD. Unlike the MCCB for schizophrenia, there remains no consensus regarding outcome measures if cognitive trials were to be undertaken in BPD. The utility of the MCCB in BPD has not yet been systematically investigated. We administered the MCCB to 80 bipolar I patients; 37 were strictly euthymic and 43 were symptomatic. We compared their performance with a demographically matched healthy sample $(n=148)$ on seven MCCB domains, and the composite. BPD patients were statistically significantly impaired on five of seven MCCB domains at levels consistent with meta-analytic studies of cognition in BPD. In contrast, patients' performance was less impaired on the Reasoning and Problem-solving and Social Cognition domains, differences that did not survive statistical correction for multiple testing. Symptomatic status only modestly influenced performance. These data suggest that the MCCB, devised for use in schizophrenia, may also represent a useful outcome measure in cognitive trials for BPD. Additional studies should address important psychometric features such as repeatability and potential practice and/or ceiling effects. Neuropsychopharmacology (20I I) 36, 1587-1592; doi:I0.1038/npp.201 I.36; published online 30 March 20I I
\end{abstract}

Keywords: bipolar I disorder; neurocognition; MATRICS; schizophrenia; psychometric; euthymic

\section{INTRODUCTION}

Neurocognitive impairment is common in bipolar disorder (BPD). Cognitive deficits, most notably in attention, verbal learning, and executive function can be observed across multiple phases of BPD, with deficits during acute episodes that are comparable in severity to those reported in schizophrenia. (Daban et al, 2006; Martinez-Aran et al, $2000,2004)$ Several meta-analyses indicate that the deficits during affective remission tend to be less severe than those noted in schizophrenia; however, performance still falls $\frac{3}{4}$ to $1 \mathrm{SD}$ below that of healthy comparison samples (Arts et al, 2008; Bora et al, 2009a; Robinson et al, 2006). Persistent deficits may contribute significantly to functional disability in BPD (Bowie et al, 2010; Burdick et al, 2010; Jaeger et al,

*Correspondence: Dr KE Burdick, The Zucker Hillside Hospital; 75-59 263rd Street, Glen Oaks, NY I I004, USA, Tel: + I 7184708167 , Fax: + I 718343 1659, E-mail: kburdick@nshs.edu

Received 14 December 2010; revised 3 February 201 I; accepted 23 February 2011
2007; Malhi et al, 2007; Martinez-Aran et al, 2007), making them an important target for future treatment (Burdick et al, 2007). Unlike a more developed approach in schizophrenia, there remains no consensus on the optimal outcome measures for clinical trials targeting cognition in BPD.

\section{MATRICS for Schizophrenia}

The Measurement and Treatment Research to Improve Cognition in Schizophrenia (MATRICS) initiative, funded by the National Institute on Mental Health, was a collaborative effort among the academia, the United States Food and Drug Administration (FDA), and the pharmaceutical industry designed to support the development of pharmacological agents to treat cognitive deficits in schizophrenia (http://www.matricsinc.org) (Green and Nuechterlein, 2004). The first step in the MATRICS process was to identify a neurocognitive battery that would be most useful for clinical trials targeting cognition in schizophrenia patients, which resulted in the final product, the MATRICS Consensus Cognitive Battery (MCCB) (Nuechterlein et al, 
2008). The MCCB has been acknowledged by the US FDA as the accepted battery for industry-based registry studies striving for an indication for cognitive enhancement in schizophrenia, and has demonstrated excellent reliability and small practice effects in large multi-site trials. (Buchanan et al, 2010; Keefe et al, 2010) Whether this battery also represents an appropriate outcome for BPD trials has not been systematically investigated; however, given the past course of drug development, evaluation, and marketing, it is likely that any compound shown to have cognitive benefits in schizophrenia will subsequently be tested in BPD. There may well be additional or alternative neurocognitive measures better suited to BPD than the MCCB, but cognitive trials expanding from schizophrenia to BPD will probably utilize the same primary outcome measures, making the evaluation of the MCCB in BPD an important first step. Although the MCCB has now been implemented internationally in several schizophrenia studies, there are no published data on the utility of this battery in BPD patients.

Therefore, we have administered the MCCB to a cohort of bipolar I patients as a first step toward evaluating its appropriateness for future clinical trials in BD. As the MCCB was designed specifically for use in schizophrenia trials, we sought to determine whether the MCCB is sensitive to the more subtle deficits noted in BPD.

\section{SUBJECTS AND METHODS}

\section{Subjects}

The study group included 80 patients with bipolar I disorder (55.0\% female; $56.3 \%$ Caucasian) with a mean age of $39.8 \pm 11.2$ years, and an estimated IQ (based on WRAT-3 Reading) of $97.4 \pm 10.3$. All subjects provided written informed consent to an Institutional Review Board of the North Shore-Long Island Jewish Health System (NSLIJHS)-approved protocol. Patients were recruited from the outpatient department of the Zucker Hillside Hospital, a division of the NSLIJHS, in Glen Oaks, NY.

All patients were clinically stable outpatients at the time assessment, with a mean Hamilton Depression Rating Scale (HDRS) total score of $10.5 \pm 7.9$ and a Clinician-Administered Rating Scale for Mania (CARS-M) total score of $6.0 \pm 7.0$ at the time of testing. A total of 58 of 80 patients $(72.5 \%)$ had a psychosis history over the course of their illness. The average age at onset of the first episode of either polarity was $22.40 \pm 8.7$ years and, on average, patients had experienced $3.67 \pm 3.1$ previous depressive episodes and $3.84 \pm 3.9$ previous manic episodes.

A total of 37 of the 80 patients met strict criteria for euthymia, based on a HDRS score of $\leqslant 8$ and a CARS-M score of $\leqslant 8$, whereas the remaining 43 patients were categorized as symptomatic. Given previous evidence suggesting that both acute and subclinical affective symptoms can significantly influence cognitive performance (Quraishi and Frangou, 2002), we included symptomatic status in secondary statistical analyses. All bipolar subjects were taking psychotropic medications; however, because of the heterogeneity of treatment, we were unable to fully control for medication status in our analyses. We did conduct exploratory analyses incorporating classes of medications.
Healthy control subjects $(n=148)$ were recruited from the general population via word of mouth, newspaper and Internet advertisements, and posted flyers. Subjects were excluded if they had an Axis I diagnosis, active or recent substance abuse, or if they had a history of CNS trauma, neurological disorder, or previously diagnosed learning disability. Controls were $43.9 \%$ female, $47.3 \%$ Caucasian, with a mean age of $41.6 \pm 15.1$ years, and an estimated IQ (based on WRAT-3 Reading) of $102.8 \pm 8.9$.

\section{Diagnostic and Clinical Measures}

Patient diagnosis was established through a structured interview (Structured Clinical Interview-DSM-IV; SCID-IV) (First et al, 2002) and confirmed by diagnostic consensus conference, which utilizes expert clinical opinion alongside SCID-IV data and corroborating medical record information. Healthy controls for the project were assessed using the SCID-IV, non-patient edition, specifically designed for healthy subjects to rule out Axis I diagnoses. Affective symptom severity was evaluated at the time of testing using the HDRS and the CARS-M rating scales administered by highly trained psychometricians with excellent inter-rater reliability $(\mathrm{ICC}>0.80)$.

\section{Neurocognitive Assessment: the MCCB}

The MATRICS committee identified seven cognitive domains that are reliably impaired in schizophrenia (Nuechterlein et al, 2004) and chose 10 standardized cognitive measures to be included in the battery (Green and Nuechterlein, 2004) after evaluating each for their test-retest reliability, utility as a repeated measure, practicality, and tolerability (Nuechterlein et al, 2008). The final MCCB battery requires approximately 70 min to administer and it consists of: Trail Making Test Part A; Brief Assessment of Cognition in Schizophrenia: Symbol coding; Hopkins Verbal Learning Test (HVLT); Wechsler Memory Scale Spatial span; Letter-number Span; Neuropsychological Assessment Battery (NAB): Mazes; Brief Visuospatial Memory Test; Category fluency; Mayer-Salovey-Caruso Emotional Intelligence Test (MSCEIT): Managing Emotions; and the Continuous Performance Test: Identical Pairs. (Nuechterlein et al, 2008). The seven MCCB domains are: Speed of Processing, Attention/Vigilance, Working Memory, Verbal Learning, Visual Learning, Reasoning/Problem-solving, and Social Cognition. The MCCB has previously been administered to 300 community volunteers at five US sites for co-norming and standardization (Kern et al, 2008). Each participant in the current study completed the full MCCB in one visit.

\section{Statistics}

Although published MCCB normative data are available as a reference group (Kern et al, 2008), our healthy control sample was better matched to our bipolar patients on demographic features. Specifically, the published norms are stratified by age and gender but not by race or ethnicity, and the Kern et al (2008) sample is characterized as $76 \%$ Caucasian, whereas our patient group is only $56 \%$ Caucasian. Therefore, we calculated $Z$-scores based on means and SDs, derived from our own healthy control data, which were better matched across all demographic features, 
Table I MCCB Performance by Group Using the MCCB Normative Data for Standardization

\begin{tabular}{|c|c|c|}
\hline $\begin{array}{l}\text { MCCB } \\
\text { Domain }\end{array}$ & $\begin{array}{l}\text { Healthy controls }(n=148) \\
\text { mean } t \text {-score (SD) }\end{array}$ & $\begin{array}{c}\text { Bipolar patients }(n=80) \\
\text { mean } t \text {-score (SD) }\end{array}$ \\
\hline $\begin{array}{l}\text { Speed of } \\
\text { processing }\end{array}$ & $49.64(9.16)$ & $38.43(9.76)$ \\
\hline $\begin{array}{l}\text { Attention } \\
\text { vigilance }\end{array}$ & $48.45(9.32)$ & $37.85(12.85)$ \\
\hline $\begin{array}{l}\text { Working } \\
\text { memory }\end{array}$ & $46.22(10.12)$ & 37.85 (1 1.07) \\
\hline Verbal learning & $43.55(8.10)$ & $38.69(7.84)$ \\
\hline Visual learning & $43.55(10.02)$ & $33.96(12.00)$ \\
\hline $\begin{array}{l}\text { Reasoning } \\
\text { problem solving }\end{array}$ & $44.86(9.19)$ & 40.99 (8.73) \\
\hline Social cognition & $49.29(10.70)$ & $44.68(12.90)$ \\
\hline Composite & $44.19(9.20)$ & $32.45(11.08)$ \\
\hline
\end{tabular}

including race. These calculations were carried out using the standardized scores from the MCCB scoring program. The MCCB automated scoring program calculates $t$-scores for each MCCB domain using the published normative sample from Kern et al (2008). Specifically, cognitive domain scores that include multiple measures are calculated by summing the $t$-scores of the tests included in that domain and then standardizing the sum to a $t$-score based on the community sample (Kern et al, 2008). The MCCB composite score was calculated in the same manner. Because we opted to utilize our own healthy comparison sample, for descriptive purposes and for comparison with other studies that have utilized the published normative data, we report in Table 1 the mean $t$-scores for our healthy and bipolar samples as compared with MCCB normative data (mean $=50 ; \mathrm{SD}=10)$.

For all further analyses, we utilized our own healthy control data and we tested for group differences on the seven cognitive domains of the MCCB plus the MCCB composite score using a multivariate analyses of covariance with subject type (bipolar $v s$ control) as a fixed factor and including WRAT-3 Reading scores (estimated premorbid IQ) as a covariate. Effect sizes are represented along the $Z$-score scale with a mean of zero and a $\mathrm{SD}$ of one. Bonferroni correction was applied to account for multiple testing.

Secondary analyses using the same approach included three groups: healthy controls $n=148$ ), symptomatic bipolar patients $(n=43)$, and euthymic bipolar patients $(n=37)$ to allow us to address the effects of symptom severity on MCCB performance.

Medications were coded dichotomously (yes/no) on the basis of four psychotropic classes: lithium, antidepressants, anticonvulsants, and antipsychotics; MANCOVAs were conducted to determine the influence of medications on MCCB performance after controlling for premorbid IQ and current mood symptoms (HRSD and CARS-M) in the bipolar sample.

\section{RESULTS}

Bipolar patients did not differ significantly from healthy controls on age, sex, or race distribution (all $p$-values $>0.11$ ); however, premorbid IQ estimated from WRAT

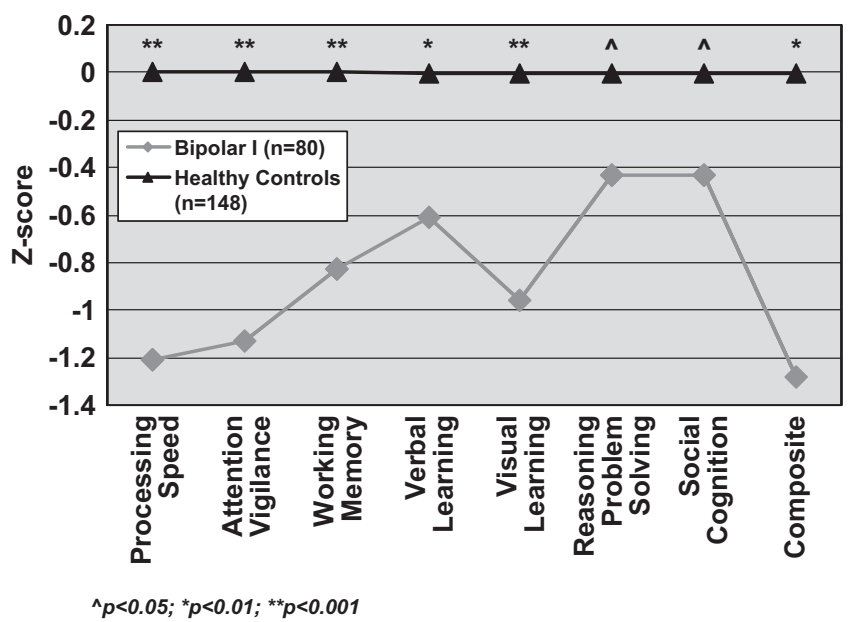

Figure I MCCB performance in 80 bipolar I patients vs 148 healthy controls: The $x$ axis indicates the seven MCCB domains and the composite score. The $y$ axis depicts a $Z$-scale score with a mean of zero and a SD of I. After correction for multiple testing, bipolar patients perform significantly worse than healthy subjects on five of seven domains and the composite score. Performance was impaired on all seven domains when using an uncorrected $p$-value $<0.05$.

reading scores was higher in the controls than in the bipolar patients $(t=4.13, \mathrm{df}=226, p<0.001)$ and this was incorporated as a covariate in all subsequent analyses. Multivariate analysis of covariance revealed a highly significant overall group effect (General Linear Model $\mathrm{F}=10.86$, Wilks' Lambda $\left.=0.72 ; \mathrm{df}=8,218, p=7.6 \times 10^{-13}\right)$ and significant group differences on all seven MCCB domains, and on the MCCB composite score such that bipolar patients performed significantly worse than healthy controls (Figure 1). After Bonferroni correction $(0.05 / 8=0.006)$, two of the seven domain scores were no longer significantly different between groups (Reasoning and Problem-solving and Social Cognition).

A second MANCOVA was conducted to better elucidate the effects of mood symptoms on MCCB performance in the BPD cohort. The symptomatic $(n=43)$ patients did not differ from the euthymic group $(n=37)$ on age $(\mathrm{F}=0.15$; $\mathrm{df}=1,78 ; p=0.70)$, sex distribution $\left(\chi^{2}=0.55 ; \mathrm{df}=1\right.$; $p=0.46)$, or race distribution $\left(\chi^{2}=2.97 ; \mathrm{df}=1 ; p=0.09\right)$. There was also no patient group difference with regard to psychosis history $\left(\chi^{2}=0.35 ; \mathrm{df}=1 ; p=0.56\right)$. The MANCOVA again revealed a highly significant effect of group (BPD symptomatic; BPD euthymic; Healthy control) (General Linear Model $\mathrm{F}=5.99$; Wilks' Lamda $=0.67 ; \mathrm{df}=16$, $434 ; p=6.03 \times 10^{-12}$ ). Five of the seven domains plus the composite score were significantly different by group; Reasoning and Problem-solving and Social Cognition were no longer significant in this model. Post hoc testing revealed that the symptomatic BPD patients were significantly impaired relative to the healthy controls on all seven domains and the composite score. In contrast, the euthymic BPD group was significantly impaired relative to healthy controls on five domains and the composite, again with the exception of the Reasoning and Problem-solving and the Social Cognition domains (Table 2). When comparing symptomatic BPD and euthymic BPD patients with each other, there were no significant group differences noted 
Table 2 Group Performance on the MCCB: Symptomatic vs Euthymic Bipolar Patients Relative to Healthy Controls (Z-scores)

\begin{tabular}{|c|c|c|c|c|c|c|}
\hline MCСB Domain & $\begin{array}{c}\text { Healthy } \\
(n=148) \text { mean (SD) }\end{array}$ & $\begin{array}{c}\text { BPD Euthymic } \\
(n=37) \text { mean (SD) }\end{array}$ & $\begin{array}{c}\text { BPD with Sx } \\
(n=43) \text { mean (SD) }\end{array}$ & $\begin{array}{l}\text { HC vs BPD } \\
\text { euthymic F (p) }\end{array}$ & $\begin{array}{l}\text { HC vs BPD } \\
\text { with Sx F (p) }\end{array}$ & $\begin{array}{l}\text { BPD Euthymic vs } \\
\text { BPD with Sx F (p) }\end{array}$ \\
\hline Speed of processing & $0.01(1.0)$ & $-1.19(1.0)$ & $-1.23(1.2)$ & $6.60(<0.00 \mathrm{I})$ & $6.91(<0.001)$ & $0.17(0.87)$ \\
\hline Working memory & $0.00(1.0)$ & $-0.98(1.1)$ & $-0.69(1.1)$ & $5.27(<0.00 \mathrm{I})$ & $3.91(<0.001)$ & $1.19(0.24)$ \\
\hline Verbal learning & $-0.01(1.0)$ & $-0.61(0.9)$ & $-0.60(1.0)$ & $3.35(0.00 \mathrm{I})$ & $3.43(0.001)$ & $0.07(0.95)$ \\
\hline Social cognition & $-0.00(1.0)$ & $-0.24(1.3)$ & $-0.60(1.1)$ & I.0। (0.32) & $3.40(0.001)$ & $1.33(0.19)$ \\
\hline Composite & $-0.00(1.0)$ & $-1.27(1.2)$ & $-1.28(1.2)$ & $6.60(<0.001)$ & $7.06(<0.001)$ & $0.05(0.96)$ \\
\hline
\end{tabular}

on any of the MCCB domain scores (Table 2; all p-values $>0.19$ ).

\section{Medications}

Approximately $50 \%$ of the subjects were prescribed anticonvulsant mood stabilizers; $80 \%$ were taking antipsychotic medications; $33 \%$ were prescribed lithium; and 31\% were taking an antidepressant at the time of testing. After controlling for current mood symptom severity and premorbid IQ, there were no significant differences on any of the MCCB domains or composite when comparing patients on/off lithium $(\mathrm{F}=1.17 ; \mathrm{df}=79 ; p=0.33)$, anticonvulsants $(\mathrm{F}=1.46 ; \mathrm{df}=79 ; \quad p=0.19)$, antipsychotics $(\mathrm{F}=1.17 ; \mathrm{df}=79 ; p=0.33)$, or antidepressants $(\mathrm{F}=0.43$; $\mathrm{df}=79 ; p=0.90)$.

\section{DISCUSSION}

In the current study, we evaluated neurocognitive performance in a cohort of bipolar I patients using the MCCB, a battery initially devised for use in clinical trials targeting cognition in patients with schizophrenia. This study is the first to report on the MCCB in a clinical sample other than schizophrenia and the results indicate that the MCCB adequately captures the most common deficits reported in BPD. Specifically, statistically significant impairments were present in the BPD patients in the domains of processing speed, attention, working memory, verbal learning, and visual learning; whereas certain executive functions (Reasoning and Problem-solving) and social cognition were not statistically significantly impaired. The severity of the impairments noted in our euthymic group were comparable to those previously reported by meta-analyses with effect sizes ranging from -0.6 to $1.2 \mathrm{SDs}$ below average (Arts et al, 2008; Bora et al, 2009a; Robinson et al, 2006; Torres et al, 2007).

Of note, the symptomatic status of the BPD groups only modestly influenced the degree of impairment detected for most of the MCCB domains. There were no significant differences between euthymic BPD patients and symptomatic BPD patients on any of the MCCB domain scores; however, although symptomatic patients showed significant impairment on Reasoning and Problem-solving and Social
Cognition, euthymic BPD patients did not significantly differ from the healthy controls in these domains. Future studies with larger samples using longitudinal designs will be necessary to better understand the effects of acute and subclinical affective symptoms on cognition within subjects, as the degree of cognitive recovery during affective remission is likely to be somewhat heterogeneous in $\mathrm{BD}$ (Bora et al, 2010).

In a recent review, Yatham et al (2010) rated the potential utility of different MCCB subtests in patients with BPD. Many of the MCCB subtests were considered to be highly applicable to bipolar samples based on previous research; however, several received reduced ratings based on limited available data. One such task, the HVLT has not been widely used in previous BPD studies, most of which have utilized a more challenging memory test (California Verbal Learning Test (CVLT)). The effect size reported in this paper for the euthymic BPD subjects in the verbal learning domain (Cohen's $D=-0.64$ ) is somewhat smaller than those reported in meta-analytic studies using the CVLT (Cohen's $D=-0.83$ ) (Bora et al, 2009a). This smaller effect size is consistent with a previous report (Schretlen et al, 2007), suggesting that this is not specific to our sample but rather reflects a slightly reduced sensitivity of the HVLT as compared with CVLT (Lacritz and Cullum, 1998) to detect the full degree of impairment in bipolar patients. Still, for use in clinical trials in which repeated testing is inherent, the HVLT is likely to maintain an advantage over the CVLT because of the availability of many alternate equivalent forms. Moreover, Yatham et al, (2010) rated the NAB Mazes and MSCEIT subtests as having 'unclear' utility because of lack of published data on these measures in BPD. It is interesting that these are the two tasks/domains that we did not detect significant deficits in the euthymic bipolar sample. Executive functioning deficits in BPD patients are reported on some (Trailmaking Test Part B $(D=0.86)$; Wisconsin Card Sorting Test (WCST; $D=0.70$ )) (Bora et al, 2009a), but not all, executive tasks. Thus, it is likely that NAB Mazes does not adequately assay the domain of Reasoning and Problem-solving in BPD, whereas a task such as the WCST might be more sensitive. Again, in the context of clinical trials for cognition, other test criteria must be considered including practice effects and alternate forms; therefore, although the WCST may tap into a different executive process, it still may not represent an 
optimal outcome measure for cognitive enhancement trials in BPD. Finally, research on social cognition in BPD is limited. Several studies have demonstrated impaired performance on some theory of mind (ToM) tasks in BPD that might assay a different construct than the MSCEIT; however it is unclear that to which extent ToM deficits are a byproduct of impaired executive functions (Bora et al, 2009b).

Although BPD patients have a similar cognitive profile when compared qualitatively with schizophrenia patients, the deficits are generally less severe (Tabares-Seisdedos et al, 2008) and there are several features of illness that influence cognitive functioning that differentiate BPD from schizophrenia. The episodic nature of BPD requires that studies account for current mood symptoms and clinical course factors, including number of previous episodes, age at onset, and duration of illness, bears directly on cognitive functioning (Martinez-Aran et al, 2004; Robinson and Ferrier, 2006). In addition, psychosis is present in a subgroup of approximately $50-60 \%$ of BPD I patients during acute affective episodes, whereas nearly as many BPD I patients never experience psychosis at all (APA (DSM-IV-TR), 2000). Data indicate that BPD I patients with a history of psychosis consistently perform worse than BPD patients who never experience psychosis, even during affective remission (Bora et al, 2009a; Martinez-Aran et al, 2008).

Given these BPD-specific features of cognition, it is likely that the optimal neurocognitive battery for many studies of $\mathrm{BPD}$, including genetic and neuroimaging paradigms aimed at understanding its pathophysiology, would not be restricted to the MCСB. It is also likely that clinical trials will be carried forward from schizophrenia directly to a BPD sample in the case that an intervention is deemed successful for cognitive enhancement in schizophrenia. Thus, it is important to address whether the MCCB is sensitive to the more subtle impairments in BPD before it is applied in the context of a clinical cognitive enhancement trial. Our data suggest that, at least upon initial exposure to the MCCB, the deficits detected in bipolar patients are significant enough to suggest that there is room for improvement if a successful cognitive enhancement agent was to be administered.

Although the current study has taken the first step in evaluating the sensitivity of the MCCB in BPD, its appropriateness in a clinical trials design, which will necessitate repeated exposure to the battery, has not been addressed here. Additional data will be needed to evaluate the MCCB's test-retest reliability (repeatability), practice effects, and its potential ceiling effects in BPD before its use in clinical trials is justified. In addition, the relationship between the MCCB outcome measures and everyday functioning will be an area of interest for future trials (Harvey et al, 2010), given the stipulation that the US FDA will require cognitive enhancement trials to not only show evidence of cognitive benefit on the MCCB but also an improvement on co-primary measures of functional capacity.

Our study has a number of limitations. Although the cohort was relatively large, we were only able to differentiate groups based on those currently experiencing clinically significant affective symptoms $v s$ patients who were euthymic at the time of testing. We were able to obtain an estimate of the influence of these symptoms on cognitive performance; however, we could neither differentiate mania from depression nor acute from subsyndromal level pathology. Moreover, our sample had a higher rate of psychosis history $(72.5 \%)$ when compared with population estimates in bipolar I disorder (50-60\%). This composition may have resulted in an overestimation of the degree of impairment common to a more typical cohort of bipolar I subjects, as it is known that psychosis history has a deleterious influence on cognition in BPD (Bora et al, 2009a; Martinez-Aran et al, 2008). The limited number of euthymic patients with no psychosis history in our sample $(n=9)$ did not allow for a meaningful comparison in this study. The effects of the course of illness on cognitive performance, including number of previous episodes and age at onset, should be a focus of future studies with larger samples. Finally, all but two, of the patients were taking psychotropic medications at the time of evaluation. The effects of medication were assessed based on dichotomous grouping of patients either taking or not taking a specific drug class. We did not detect significant medication effects using this approach; however, it remains possible that medications commonly used to treat BPD may contribute to cognitive impairment.

We present the first report of the MCCB in a wellcharacterized sample of patients with bipolar I disorder. Our findings provide evidence supporting the potential use of this battery as a platform in future clinical trials targeting cognition in BPD. The MCCB appears to adequately assay cognitive functioning in patients with BPD, regardless of clinical state at the time of testing; however, several additional psychometric properties should be addressed before its acceptance as the primary outcome measure in such trials. Finally, extension of this work into a broader cohort of bipolar patients including non-psychotic bipolar I and bipolar II patients will be useful in understanding differences in cognitive profiles among these subtypes.

\section{ACKNOWLEDGEMENTS}

This study was financially supported by NIH K23MH077807 (to KEB). We wish to acknowledge Nisali Gunawardane, Yaniv Shaya, Nisha Chitkara, Anu Tyagi, Denise Coscia, Gail Reiter, Brady Berman, and James Riley for their contributions toward the data collection for this study.

\section{DISCLOSURE}

Dr Burdick has served on the speaker's bureau for Merck. Dr Goldberg has consulted for Merck and GSK over the past year, receives royalties for use of the BACS in clinical trials from Neurocog Trials, and was awarded an investigator-initiated grant from Pfizer. Dr Cornblatt has received investigator-initiated research funding support from notfor-profit entities including the National Institute of Mental Health and the Robert Wood Johnson Foundation. She has also served as a consultant for Eli Lilly and Company, Bristol-Myers Squibb, and Janssen Pharmaceuticals, and has received unrestricted educational grants from Janssen Pharmaceuticals. Dr Cornblatt currently serves as a consultant for Bristol-Myers Squibb and has also consulted 
for Merck during the past year. She has intellectual property rights for the CPT-IP, which is included in the MATRICS battery, and is also sold to other researchers worldwide for use in their research projects. Dr Keefe reports that he currently or in the past 2 years has received investigatorinitiated research funding support from the National Institute of Mental Health, Allon, Astra-Zeneca, GlaxoSmithKline, Novartis, Department of Veteran's Affairs, and the Singapore National Medical Research Council. He currently or in the past 2 years has received honoraria, served as a consultant, or advisory board member for Abbott, BiolineRx, BrainCells, BMS, CHDI, Cypress Bioscience, Dainippon Sumitomo Pharma, Eli Lilly, EnVivo, Lundbeck, Merck, Memory, Neurosearch, Orion, Otsuka, Pfizer, Prophase, Roche, Sanofi-Aventis, Shire, Solvay, Sunovion, Takeda, and Wyeth. Dr Keefe receives royalties from the Brief Assessment of Cognition in Schizophrenia (BACS) testing battery and the MATRICS Battery (BACS Symbol Coding). He is also a shareholder in NeuroCog Trials Drs Gopin, DeRosse, and Braga have nothing to disclose. Dr Malhotra has served as consultant or speaker for Bristol-Myers Squibb, Merck, Astra-Zeneca, Vanda Pharmaceuticals and Clinical Data and has received research support from Pfizer, Janssen Pharmaceuticals, Bristol-Myers Squibb, and Eli Lilly.

\section{REFERENCES}

Arts B, Jabben N, Krabbendam L, van Os J (2008). Meta-analyses of cognitive functioning in euthymic bipolar patients and their first-degree relatives. Psychol Med 38: 771-785.

Bora E, Yucel M, Pantelis C (2009a). Cognitive endophenotypes of bipolar disorder: a meta-analysis of neuropsychological deficits in euthymic patients and their first-degree relatives. $J$ Affect Disord 113: 1-20.

Bora E, Yucel M, Pantelis C (2009b). Theory of mind impairment: a distinct trait-marker for schizophrenia spectrum disorders and bipolar disorder? Acta Psychiatr Scand 120: 253-264.

Bora E, Yucel M, Pantelis C (2010). Cognitive impairment in affective psychoses: a meta-analysis. Schizophr Bull 36: 112-125.

Bowie CR, Depp C, McGrath JA, Wolyniec P, Mausbach BT, Thornquist $\mathrm{MH}$ et al. (2010). Prediction of real-world functional disability in chronic mental disorders: a comparison of schizophrenia and bipolar disorder. Am J Psychiatry 167: 1116-1124.

Buchanan RW, Keefe RS, Umbricht D, Green MF, Laughren T, Marder SR (2010). The FDA-NIMH-MATRICS Guidelines for clinical trial design of cognitive-enhancing drugs: what do we know 5 years later? Schizophr Bull (e-pub ahead of print).

Burdick KE, Braga RJ, Goldberg JF, Malhotra AK (2007). Cognitive dysfunction in bipolar disorder: future place of pharmacotherapy. CNS Drugs 21: 971-981.

Burdick KE, Goldberg JF, Harrow M (2010). Neurocognitive dysfunction and psychosocial outcome in patients with bipolar I disorder at 15-year follow-up. Acta Psychiatr Scand 122: 499-506.

Daban C, Martinez-Aran A, Torrent C, Tabares-Seisdedos R, Balanza-Martinez V, Salazar-Fraile J et al. (2006). Specificity of cognitive deficits in bipolar disorder versus schizophrenia. a systematic review. Psychother Psychosom 75: 72-84.

First MB, Spitzer RL, Gibbon M, Williams JBW (2002). Structured Clinical Interview for DSM-IV-TR Axis I Disorders, Research Version Patient Edition (SCIDI/P), Biometrics Research, New York State Psychiatric Institute: New York.

Green MF, Nuechterlein KH (2004). The MATRICS initiative: developing a consensus cognitive battery for clinical trials. Schizophr Res 72: 1-3.
Harvey PD, Wingo AP, Burdick KE, Baldessarini RJ (2010). Cognition and disability in bipolar disorder: lessons from schizophrenia research. Bipolar Disord 12: 364-375.

Jaeger J, Berns S, Loftus S, Gonzalez C, Czobor P (2007). Neurocognitive test performance predicts functional recovery from acute exacerbation leading to hospitalization in bipolar disorder. Bipolar Disord 9: 93-102.

Keefe RS, Fox KH, Harvey PD, Cucchiaro J, Siu C, Loebel A (2010). Characteristics of the MATRICS consensus cognitive battery in a 29-site antipsychotic schizophrenia clinical trial. Schizophr Res 125: 161-168.

Kern RS, Nuechterlein KH, Green MF, Baade LE, Fenton WS, Gold JM et al. (2008). The MATRICS consensus cognitive battery, part 2: co-norming and standardization. Am J Psychiatry 165: $214-220$.

Lacritz LH, Cullum CM (1998). The hopkins verbal learning test and CVLT: a preliminary comparison. Arch Clin Neuropsychol 13: $623-628$

Malhi GS, Ivanovski B, Hadzi-Pavlovic D, Mitchell PB, Vieta E, Sachdev P (2007). Neuropsychological deficits and functional impairment in bipolar depression, hypomania and euthymia. Bipolar Disord 9: 114-125.

Martinez-Aran A, Torrent C, Tabares-Seisdedos R, Salamero M, Daban C, Balanza-Martinez V et al (2008). Neurocognitive impairment in bipolar patients with and without history of psychosis. J Clin Psychiatry 69: 233-239.

Martinez-Aran A, Vieta E, Colom F, Reinares M, Benabarre A, Gasto C et al (2000). Cognitive dysfunctions in bipolar disorder: evidence of neuropsychological disturbances. Psychother Psychosom 69: 2-18.

Martinez-Aran A, Vieta E, Reinares M, Colom F, Torrent C, Sanchez-Moreno J et al (2004). Cognitive function across manic or hypomanic, depressed, and euthymic states in bipolar disorder. Am J Psychiatry 161: 262-270.

Martinez-Aran A, Vieta E, Torrent C, Sanchez-Moreno J, Goikolea JM, Salamero M et al (2007). Functional outcome in bipolar disorder: the role of clinical and cognitive factors. Bipolar Disord 9: $103-113$.

Nuechterlein KH, Barch DM, Gold JM, Goldberg TE, Green MF, Heaton RK (2004). Identification of separable cognitive factors in schizophrenia. Schizophr Res 72: 29-39.

Nuechterlein KH, Green MF, Kern RS, Baade LE, Barch DM, Cohen JD et al (2008). The MATRICS consensus cognitive battery, part 1: test selection, reliability, and validity. $A m \mathrm{~J}$ Psychiatry 165: 203-213.

Quraishi S, Frangou S (2002). Neuropsychology of bipolar disorder: a review. J Affect Disord 72: 209-226.

Robinson LJ, Ferrier IN (2006). Evolution of cognitive impairment in bipolar disorder: a systematic review of cross-sectional evidence. Bipolar Disord 8: 103-116.

Robinson LJ, Thompson JM, Gallagher P, Goswami U, Young AH, Ferrier IN et al. (2006). A meta-analysis of cognitive deficits in euthymic patients with bipolar disorder. J Affect Disord 93: 105-115.

Schretlen DJ, Cascella NG, Meyer SM, Kingery LR, Testa SM, Munro CA et al (2007). Neuropsychological functioning in bipolar disorder and schizophrenia. Biol Psychiatry 62: 179-186.

Tabares-Seisdedos R, Balanza-Martinez V, Sanchez-Moreno J, Martinez-Aran A, Salazar-Fraile J, Selva-Vera G et al (2008). Neurocognitive and clinical predictors of functional outcome in patients with schizophrenia and bipolar I disorder at one-year follow-up. J Affect Disord 109: 286-299.

Torres IJ, Boudreau VG, Yatham LN (2007). Neuropsychological functioning in euthymic bipolar disorder: a meta-analysis. Acta Psychiatr Scand Suppl 434: 17-26.

Yatham LN, Torres IJ, Malhi GS, Frangou S, Glahn DC, Bearden CE et al (2010). The International Society for Bipolar DisordersBattery for Assessment of Neurocognition (ISBD-BANC). Bipolar Disord 12: 351-363. 